\title{
Resource Estimation in T20 Cricket
}

\author{
Harsha Perera and Tim B. Swartz *
}

\begin{abstract}
This paper investigates the suitability of the Duckworth-Lewis method as an approach to resetting targets in interrupted T20 cricket matches. Whereas the Duckworth-Lewis method has been adopted in both international T20 matches and in the Indian Premier League, there has been growing objections to its use in T20. In this paper, we develop methodology for the estimation of a resource table designed for T20 cricket. The approach differs from previous analyses in the literature by considering an enhanced dataset. It is suggested that there exist meaningful differences in the scoring patterns between one-day cricket and T20.
\end{abstract}

Keywords: Constrained estimation, Duckworth-Lewis method, Gibbs sampling, T20 cricket.

${ }^{*}$ Harsha Perera is a Phd candidate and Tim Swartz is Professor, Department of Statistics and Actuarial Science, Simon Fraser University, 8888 University Drive, Burnaby BC, Canada V5A1S6. Swartz has been partially supported by grants from the Natural Sciences and Engineering Research Council of Canada. The authors are appreciative of the comments provided by two anonymous referees. 


\section{INTRODUCTION}

In 2003, the most recent version of cricket known as T20 (or Twenty20) was introduced. The matches were contested between English and Welsh domestic sides in what was known as the Twenty20 Cup.

Since its inception, T20 cricket has exploded in popularity. At an international level, three T20 World Cups have been constested with India, Pakistan and England prevailing in the years 2007, 2009 and 2010 respectively. Although international T20 cricket matches take place on a regular basis, the Indian Premier League (IPL) has been the major source of growth and excitement with respect to T20 cricket. The IPL has completed four successul seasons (2008, 2009, 2010 and 2011). Apart from the second season which was hosted in South Africa, IPL matches are played in major centres spread across India. In the 2011 season, the IPL expanded from 8 to 10 teams where special considerations were given (e.g. retention of players) to fuel regional support. In addition, team salary constraints have been introduced to maintain competitive balance, and the league has been promoted through its ties with Bollywood ownership and support. An IPL season typically begins in the spring (March or April) and is completed within six weeks time. The accelerated pace of the IPL season allows international players to return to their home country to resume training with their home nation. The tight schedule is also believed to keep fans riveted. The IPL is so popular that all 74 matches in 2011 were televised live in Canada, a nation with a limited cricket history.

T20 is a limited overs version of cricket which is very similar to one-day cricket. The main difference is that each batting side is given 20 overs in T20 whereas 50 overs are alloted in one-day cricket. Consequently, T20 matches last roughly three hours in duration, a time more in keeping with the popular professional sports of football, soccer, rugby, baseball, basketball and hockey. Another consequence of the reduced overs in T20 is that the style of 
batting is more aggressive. Batsmen are less concerned with dismissals as the 10 wickets are more easily preserved over fewer balls (i.e. overs).

One of the carryovers of one-day cricket to T20 has been the adoption of the DuckworthLewis method. In interrupted matches, particularly rain-interrupted matches, sometimes matches need to be shortened. In doing so, the target for the team batting second may need to be adjusted as the team batting second may not be provided the same number of overs as the team batting first. The method of resetting the target is known as the Duckworth-Lewis method.

The Duckworth-Lewis method (Duckworth and Lewis, 1998, 2004) was developed for oneday cricket based on a large-scale analysis of scoring patterns observed in one-day cricket. It is natural to ask whether the scoring patterns are the same in one-day cricket and T20. Equivalently, one may ask whether the Duckworth-Lewis method is suitable for use in T20 cricket.

Initially, the use of Duckworth-Lewis methodology in T20 did not cause much of a stir. A reason for this may be that inclement weather in T20 cricket tends to lead to the cancellation rather than the shortening of matches. And in the few matches where Duckworth-Lewis was applied, the targets appeared reasonable. However, over time, discontent has grown with various applications of Duckworth-Lewis in T20. For example,

- May 3, 2010: On Duckworth-Lewis applied in the 2010 World Cup match between West Indies and England, captain Paul Collingwood of England complained "Ninetyfive percent of the time when you get 191 runs on the board you are going to win the game". He then added, "There is a major problem with Duckworth-Lewis in this form of the game".

- May 5, 2010: Commenting on a variety of issues in cricket, former Pakistani bowler Abdul Qadir expressed the opinion, "One needs to revisit and rethink the D/L method 
and on top of that its use in T20 format".

- April 18, 2011: Chennai Super Kings coach Stephen Fleming on Duckworth-Lewis after loss to Kochi where Duckworth-Lewis was applied, "it is rubbish for Twenty20".

We suggest that we are now at a point in time where a sufficiently large dataset of T20 matches has accumulated to adequately assess whether there is a difference in scoring patterns between one-day cricket and T20. This paper is concerned with the comparison of scoring patterns in the two versions of cricket. Accordingly, we develop a resource table analogous to the Duckworth-Lewis resource table for the resetting of targets in interrupted T20 matches.

A T20 resource table is a matrix of the resources remaining relative to the status of a match. The table has rows corresponding to overs available, ranging from 20 to 1, and the table has columns corresponding to wickets lost, ranging from 0 to 9 . Accordingly, a T20 resource matrix should logically contain strictly decreasing entries along both the rows and the columns. Using constrained maximum likelihood estimation for the unknown cell entries, a problem is that maxima often occur along the boundaries, and this is unrealistic for resource tables. Consequently, our approach to constrained estimation is Bayesian where estimated entries correspond to posterior means. This ensures that strictly decreasing entries are obtained along both rows and columns. Other advantages of our Bayesian implementation are the simplicity of computation where we generate from straightforward full conditional distributions, the handling of missing data and the opportunity to introduce prior knowledge.

In Section 2, we review the Duckworth-Lewis method used in one-day cricket and how it is currently applied in T20 matches. We provide a list of suspicions as to why DuckworthLewis may not be appropriate for T20. In Section 3, we review aspects of Bhattacharya, Gill and Swartz (2010) which was the first attempt at assessing the suitability of DuckworthLewis in T20. We modify the Battacharya, Gill and Swartz (2010) methodology, hereafter 
referred to as BGS, and apply it to a much larger dataset than originally studied by BGS. In Section 4, we develop new methodology for the estimation of a resource table in T20 which overcomes a prominent weakness of BGS. In particular, we do not aggregrate scoring over all matches but retain the variability due to individual matches. The new approach differs from the Duckworth-Lewis construction in that it does not assume a parametric form on resources. The approach is Bayesian and is implemented using Markov chain Monte Carlo methodology with estimation on a constrained parameter space. The new resource table confirms some of the findings in BGS. We conclude with a short discussion in Section 4.

\section{REVIEW OF DUCKWORTH-LEWIS}

For resetting targets in interrupted one-day cricket matches, the Duckworth-Lewis method (Duckworth and Lewis, 1998, 2004) supplanted the method of run-rates in the late 1990's and has since been adopted by all major cricketing boards. With the sport of cricket having three billion followers worldwide, the Duckworth-Lewis method may be considered the greatest contribution to the sporting world from a mathematical, statistical and operational research perspective. What makes the acceptance of the Duckworth-Lewis method so remarkable is that the method is largely viewed by the public as a black-box procedure. The sporting world tends to like simple rules and simple statistics. From this point of view, the adoption of the Duckworth-Lewis method was a masterpiece in overcoming political hurdles. Although a number of competitors to the Duckworth-Lewis method have been proposed over the years (Clarke 1988, Christos 1998, Jayadevan 2002, Carter and Guthrie 2004), the DuckworthLewis method has passed the test of time and has been updated every four years to take into account changes in the one-day game.

The fundamental concept underlying the Duckworth-Lewis method is that of resources. 
In a one-day cricket match, the team batting first begins with 50 overs and 10 wickets at their disposal. They continue batting until either their overs are completed or 10 wickets have fallen. It is the combination of wickets and overs remaining in an innings that provides the capacity for scoring runs. The Duckworth-Lewis quantification of the combination of wickets and overs is known as resources. When innings are reduced due to a rain-interruption, then targets are reset to "fair" values based on the resources remaining. In one-day cricket, a team has $100 \%$ of its resources remaining at the beginning of its innings (50 overs and 0 wickets lost). When a team has used up all of its 50 overs, then $0 \%$ of its resources are remaining. Similarly, when 10 wickets are lost, a team has $0 \%$ of its resources remaining.

The application of the Duckworth-Lewis method to T20 cricket considers the status of a one-day cricket match when 20 overs and 10 wickets are available. At this state of a match, according to the Standard Edition of the Duckworth-Lewis table, 56.6\% of the batting team's resources remain. Therefore, the modified Duckworth-Lewis table for T20 is obtained from the original Duckworth-Lewis table by dividing each cell entry by 0.566 . In Table 1 , we provide the Duckworth-Lewis table (Standard Edition) scaled for T20. We observe that the table begins with $100 \%$ resources when 20 overs are available and 0 wickets are lost (top left hand corner). The table is monotonic decreasing in both the columns and rows indicating that resources diminish as overs are utilized and wickets are lost.

As an illustration of the use of Table 1, consider a T20 match in which the team batting first scores 150 runs in their innings. Then suppose that it rains, the game is delayed, and the team batting second is given only 12 overs for batting. It would be unfair for the target to be set at 151 runs since the team batting second has fewer overs to reach its target than the team batting first. From Table 1, we see that the team batting second has $66.4 \%$ of its resources remaining based on 12 available overs and 0 wickets lost. Therefore the target for winning is set at $150(0.664)=99.6 \rightarrow 100$ runs. 
Although the scaling of resources from one-day cricket to T20 is an intuitive and seemingly sensible procedure, there are a number of suspicions that the scoring patterns in one-day cricket and T20 may not be identical. These include:

- the powerplay in T20 occurs in the first 6 overs (comprising 30\% of the overs) whereas the initial powerplay in one-day cricket occurs in the first 10 overs (comprising $20 \%$ of the overs)

- the composition of teams may not be the same in the two forms of cricket; for example, there is some indication that the T20 game has a preference towards "heavy" hitters, those more able to hit 4's and 6's at higher rates

- the mapping of the one-day resource table to the T20 table involves different contexts; for example, when 20 overs and 10 wickets are available in one-day cricket, batsmen have reached a comfort level at this stage of a match whereas in T20, the batsmen are only beginning to adjust to the bowler, the ball and the pitch

For the various reasons listed above and more, it seems prudent to investigate the suitability of the Duckworth-Lewis method in the context of T20 cricket.

\section{REVIEW AND IMPLEMENTATION OF BGS}

In a preliminary analysis of the suitability of the Duckworth-Lewis method in T20, BGS considered first innings scoring results from $n=85$ T20 matches. The matches took place between February 17, 2005 through November 9, 2009 and involved international ties between nations belonging to the International Cricket Council (ICC). Second innings data were not used as it is well-known that second innings batting tactics depend on the state of the match and the target score. 
For each match, BGS defined $x(u, w(u))$ as the runs scored from the stage in the first innings where $u$ overs are available and $w(u)$ wickets are lost until the end of the first innings. The estimates $r_{u, w}$ were then obtained where $r_{u, w}$ is the percentage of resources remaining when $u$ overs are available and $w$ wickets are lost, $u=1, \ldots, 20$ and $w=0, \ldots, 9$. BGS calculated $r_{u, w} / 100 \%$ by averaging $x(u, w(u))$ over all matches where $w(u)=w$ and dividing by the average of $x(20,0)$ over all matches. The sample standard deviation corresponding to $r_{u, w}$ was denoted by $\sigma_{u, w}$. In the case of missing values, BGS set $r_{u, w}$ equal to the corresponding Duckworth-Lewis table entry and $\sigma_{u, w}=5.0$.

Motivated by isotonic regression in two variables, BGS then constructed a resource table consisting of the posterior means of the $y_{u, w}$ which were estimated via Gibbs sampling from the full conditional distributions

$$
\left[y_{u, w} \mid \cdot\right] \sim \operatorname{Normal}\left[r_{u, w}, \sigma_{u, w}^{2}\right]
$$

subject to $y_{20,0}=100, y_{0, w}=0, y_{u, 10}$ and the table monotonicity constraints $y_{u, w} \geq y_{u, w+1}$ and $y_{u, w} \geq y_{u-1, w}$ for $u=1, \ldots, 20$ and $w=0, \ldots, 9$. Sampling from (1) is easily carried out using a normal generator and rejection sampling according to the constraints.

Unlike the Duckworth-Lewis estimation procedure which assumes an exponential form on resources, the BGS procedure is nonparametric in the sense that no functional relationship is imposed on the $y$ 's. In addition, whereas the Duckworth-Lewis estimation procedure is based on an unlimited overs formulation involving an asymptote, the BGS procedure takes the limited overs nature of T20 into account.

We now consider the implementation of BGS on a much larger dataset. We have taken first innings data from all international matches involving ICC nations during the period February 17, 2005 through June 1, 2011. This provides 146 matches excluding the few matches whose first innings were shortened. In addition, we include the 242 first innings data corresponding to the first four IPL regular seasons and playoffs. In total, this comprehensive 
dataset provides $n=388$ matches involving T20 sides of the highest standard.

To investigate the suitability of pooling the international T20 data with the IPL data, we calculated percentages $r_{\mathrm{INT}, u, w}$ based on the 146 international matches, and percentages $r_{\mathrm{IPL}, u, w}$ based on the 242 IPL matches. We restricted our attention to the 88 pairs $\left(r_{\mathrm{INT}}, r_{\mathrm{IPL}}\right)$ where both percentages are based on at least 10 observations. As a very rough guide, we calculated independent $t$-tests between the pairs using the assumption of common variances. We observed that only four of the 88 pairs were rejected as having different means at the $5 \%$ level of significance. This provides some motivation in pooling the international T20 data with the IPL data.

We make one improvement to the BGS estimation procedure. Rather than use the estimates $r_{u, w}$ described above, we set $r_{u, w} / 100 \%$ equal to the average of $x(u, w(u))$ over all matches where $w(u)=w$ divided by the average of $x(20,0)$ in the corresponding matches. In the Appendix, we see that the proposed estimates have some advantages in terms of reduced variability. In addition, the improvement prevents non-sensical values such as $r_{19,2}=110.2$ as was reported in Bhattacharya, Gill and Swartz (2010).

Using the enhanced dataset, Table 2 provides a BGS resource table using the estimated posterior means of the $y$ 's obtained through Gibbs sampling. The computations posed no difficulties and the estimates stabilized after 50,000 iterations. There are three main discrepancies between the Duckworth-Lewis table for T20 (Table 1) and the new BGS Table 2. The first discrepancy occurs along the top of the tables where BGS values exceed the DuckworthLewis table entries. This is generally not problematic since matches rarely (never) correspond to this section of the table. However, we note that the new BGS table is nonsensical in the top right corner where $18.1 \%$ resources remain when 20 overs are available and 9 wickets are lost. Clearly, the last batsman is weaker than batsmen at the beginning of the batting order, and hence, the maximum value for that cell is $10 \%$. The second discrepancy between 
Table 1 and Table 2 occurs in the bottom right corner which is a realistic scenario. The new BGS entries in the bottom right corner are roughly $5 \%$ less than in the Duckworth-Lewis table. The third discrepancy seldomly occurs in practice and involves the last 7 overs when zero wickets are lost. Here, the new BGS table has entries that are roughly $3 \%$ larger than the Duckworth-Lewis table. With respect to the second and third discrepancies, a similar pattern was also observed in Bhattacharya, Gill and Swartz (2010). As one might expect, increasing the dataset from $n=85$ used in Bhattacharya, Gill and Swartz (2010) to $n=388$ in our new BGS implementation resulted in some improved estimates. A discussion of these improvements is provided in Perera (2011).

\section{A NEW RESOURCE TABLE FOR T20 CRICKET}

A weakness of the BGS procedure is that the observed resource percentages $r_{u, w}$ are calculated as aggregates over all matches. For the estimation of resources, it is desirable to avoid the unnecessary summarization of data, and instead account for the variability of scoring patterns with respect to individual matches. In this section, we propose a more sophisticated statistical model which utilizes the individual match data.

We vary the notation and let $r_{i}=\left(r_{i, 20}, \ldots, r_{i, n_{i}}\right)^{\prime}$ for match $i=1, \ldots, n$ where $r_{i, u}$ is the percentage of runs scored in match $i$ with $u$ overs available until the end of the first innings, $u=20,19, \ldots, n_{i}$. Note that $n_{i}>1$ implies that the batting team used up all of its wickets. For example, $r_{i, 20}=100 \%$ and $r_{i, 19}$ is the percentage of runs scored in the first innings since the end of the first over. The covariate $w_{i, u}$ is the number of wickets lost at the stage of the $i$ th match where $u$ overs are available.

Let $[A \mid B]$ generically denote the density function or probability mass function corresponding to $A$ given $B$. Then using conditional probability, the likelihood of the first innings 
data is given by

$$
\prod_{i=1}^{n}\left[r_{i, 20}, \ldots, r_{i, n_{i}}\right]=\prod_{i=1}^{n}\left[r_{i, n_{i}} \mid r_{i, n_{i}+1}\right] \cdots\left[r_{i, 19} \mid r_{i, 20}\right] .
$$

The goal is to obtain a resource table whose entries $\theta_{u, w}$ are the expected percentage of resources remaining when $u$ overs are available and $w$ wickets are lost. The percentages satisfy the constraints $\theta_{20,0}=100 \%, \theta_{0, w}=\theta_{u, 10}=0 \%, \theta_{u, w} \geq \theta_{u-1, w}$ and $\theta_{u, w} \geq \theta_{u, w+1}$, for $u=1, \ldots, 20, w=1, \ldots, 9$. Our key modelling assumption is that

$$
\left[r_{i, u} \mid r_{i, u+1}\right] \sim \operatorname{Normal}\left[r_{i, u+1}+\theta_{u, w_{i, u}}-\theta_{u+1, w_{i, u+1}}, \sigma^{2}\right]
$$

which states that the observed change in resources $r_{i, u}-r_{i, u+1}$ is centred about the expected change. As with BGS, the proposed model does not imply a functional relationship on resources; the goal is to allow the data to determine the resource percentages $\theta_{u, w}$, subject to the monotonicity constraints. It is possible to introduce a specific $\sigma$ for each pair $(u, w)$ but this essentially doubles the number of parameters from 101 to 200. Furthermore, it does not lead to appreciably improved results.

To facilitate the estimation of $\theta_{u, w}$, we consider a Bayesian approach based on Markov chain methodology where we impose a flat prior $[\theta] \propto 1$ and a standard reference prior $\left[\sigma^{2}\right] \sim$ Inverse Gamma[1.0,1.0]. A good reference on Markov chain methods is the edited text by Gilks, Richardson and Spiegelhalter (1996). Using the likelihood (2), the modelling assumption (3) and the prior specification, we obtain the full conditional distribution

$$
\left[\sigma^{2} \mid \cdot\right] \sim \text { Inverse Gamma. }\left[m_{u, w}+1,\left(m_{u, w}+2\right) / 2\right]
$$

where $m_{u, w}$ is the number of matches that pass through $(u, w)$. The remaining full conditional distributions are given by

$$
\left[\theta_{u, w} \mid \cdot\right] \sim \operatorname{Normal}\left[\tau_{u, w}, \sigma^{2} /\left(2 m_{u, w}\right)\right]
$$


subject to the constraints $\max \left(\theta_{u-1, w}, \theta_{u, w+1}\right) \leq \theta_{u, w} \leq \min \left(\theta_{u+1, w}, \theta_{u, w-1}\right)$ for $u=1, \ldots, 19$ and $w=1, \ldots, 9$, where

$$
\tau_{u, w}=\frac{1}{2 m_{u, w}} \sum\left(r_{i, u}-r_{i, u+1}+\theta_{u+1, w_{i, u+1}}+r_{i, u}-r_{i, u-1}+\theta_{u-1, w_{i, u-1}}\right)
$$

and the sum is taken over all matches $i$ that pass through $(u, w)$.

We have coded a Gibbs sampling algorithm which iteratively simulates from the full conditional distributions in (4) and (5). Using 50,000 iterations, we estimated the posterior means of the $\theta_{u, w}$ leading to the resource table shown in Table 3. This required roughly 30 minutes of computation. To help facilitate the comparison between the Duckworth-Lewis table 1 and the new Table 3, we refer to the heat map in Figure 1. The darkest shades of the heat map represent changes of roughly $8 \%$. As was found in the BGS implementation (Table 2), our new Table 3 has the greatest differences when compared to Duckworth-Lewis Table 1 along the main diagonal. In the upper-left corner, our resource table provides more resources than Duckworth-Lewis. A possible explantion for this is that the upper-left corner corresponds to the beginning of a match where batsmen are gaining familiarity with the match conditions (i.e. the bowler, the pitch, the ball). Consequently, batsmen are more cautious in the early parts of the match, and therefore few resources are utilized in this part of the table. Recall that the Duckworth-Lewis table for T20 at this stage of a match was mapped from the Duckworth-Lewis one-day table where 20 overs are available and zero wickets are lost. In one-day cricket, this corresponds to a period of more aggressive batting where the batsmen are comfortable. In the middle section of the diagonal, the new resource Table 3 has fewer resources than the Duckworth-Lewis Table 1. This departure from Duckworth-Lewis was also observed in Bhattacharya, Gill and Swartz (2010) and in the BGS implementation of Section 3 leading to Table 2. Although the shading in the lower-left hand corner of the heat map is not as intense as in the diagonal section, these differences are meaningful and are worthy of comment. In the discussion, we look back at 
matches where this section of the table was relevant. We see that the new resource Table 3 has more resources in the lower-left corner than does the Duckworth-Lewis Table 1. Finally, we observe that the upper-right hand corner of the new resourse table is in accordance with Duckworth-Lewis. This is an advantage of the new resource Table 3 over BGS (Table 3) which gave non-sensical results in the upper-right hand corner.

\section{DISCUSSION}

Before looking at real examples, we emphasize again that there is now a sufficient number of T20 matches to assess differences in the scoring patterns between one-day cricket and T20. Moreover, unlike Duckworth-Lewis, the new methodology of section 4 leading to Table 3 does not make any parametric assumptions about resources. The methodology allows the data to determine the cell entries. The methodology is also preferable to BGS in that it takes into account the variability of individual matches.

We conclude our discussion with two examples where the application of Duckworth-Lewis in T20 has been criticized.

England vs West Indies, June 15/09: England batted first and made 161 for 6 wickets in 20 overs. After a rain interruption, the West Indies innings was reduced to 9 overs. The Duckworth-Lewis method (Professional Edition) was used to reset the target to 80 runs. The West Indies achieved the target in 8.2 overs and the result was criticized on the basis of the target being too low. Using the new table 3, the target would have been 86 runs.

England vs West Indies, May 3/10: England batted first and made 191 for 5 wickets in 20 overs. After a rain interruption, the West Indies innings was reduced to 6 overs. The Duckworth-Lewis method (Professional Edition) was used to reset the target to 
60 runs. The West Indies achieved the target in 5.5 overs and the result was again criticized on the basis of the target being too low. Using the new table 3 , the target would have been 73 runs.

Finally, we state that our intention is not to supplant the Duckworth-Lewis method, but to highlight its shortcomings in T20 cricket so that it can be improved upon from the point of view of players, officials, and fans.

\section{REFERENCES}

Bhattacharya, R., Gill, P.S. and Swartz, T.B. (2010). Duckworth-Lewis and twenty20 cricket. Journal of the Operational Research Society, doi: 10.1057/jors.2010.175.

Carter, M. and Guthrie, G. (2004). Cricket interruptus: fairness and incentive in limited overs cricket matches. Journal of the Operational Research Society, 55: 822-829.

Christos, G.A. (1998). It's just not cricket. In Mathematics and Computers in Sport (N. de Mestre and K. Kumar, editors), Bond University, Queensland, Australia, pp 181-188.

Clarke, S.R. (1988). Dynamic programming in one-day cricket - optimal scoring rates. Journal of the Operational Research Society, 39: 331-337.

Duckworth, F.C. and Lewis, A.J. (1998). A fair method for resetting targets in one-day cricket matches. Journal of the Operational Research Society, 49: 220-227.

Duckworth, F.C. and Lewis, A.J. (2004). A successful operational research intervention in one-day cricket. Journal of the Operational Research Society, 55: 749-759.

Gilks, W.R., Richardson, S. and Spiegelhalter, D.J. (editors) (1996). Markov Chain Monte Carlo in Practice. Chapman and Hall: London. 
Jayadevan, D. (2002). A new method for the computation of target scores in interrupted, limited over cricket matches. Current Science, 83, 577-586.

Perera, H. (2011). A second look at Duckworth-Lewis in Twenty20 cricket. MSc project, Simon Fraser University, Department of Statistics and Actuarial Science.

\section{APPENDIX}

We consider the estimation of $r_{u, w}$ in section 3. For ease of notation, we drop the subscript $u, w$ and let the index $i=1, \ldots, m$ denote the matches which pass through the stage of the first innings where $u$ overs are available and $w$ wickets are lost. Further, let $x_{i}$ be the runs scored from that juncture in the $i$ th match until the end of the first innings, let $y_{i}$ be the total first innings runs in the $i$ th match, and let $z_{j}$ be the total first innings runs in the $j$ th match, $j=1, \ldots, n-m$ where the index $j$ corresponds to the remaining matches in the dataset. The data are of the form

$$
\left(\begin{array}{c}
x_{1} \\
y_{1}
\end{array}\right), \ldots,\left(\begin{array}{c}
x_{m} \\
y_{m}
\end{array}\right) \text { iid }
$$

with $\mathrm{E}\left(x_{i}\right)=\mu_{1}, \operatorname{Var}\left(x_{i}\right)=\sigma_{1}^{2}, \mathrm{E}\left(y_{i}\right)=\mu_{2}, \operatorname{Var}\left(y_{i}\right)=\sigma_{2}^{2}$ and $\operatorname{Cov}\left(x_{i}, y_{i}\right)=\sigma_{12}$. We assume that the correlation

$$
\frac{\sigma_{12}}{\sigma_{1} \sigma_{2}}>1 / 2
$$

reflecting the tendency that high (low) scoring matches are typically high (low) scoring throughout the match. In addition, there are independent data

$$
z_{1}, \ldots, z_{n-m} \text { iid }
$$

with $\mathrm{E}\left(z_{j}\right)=\mu_{2}$ and $\operatorname{Var}\left(z_{j}\right)=\sigma_{2}^{2}$. 
Using the above notation, the BGS estimator takes the form

$$
\hat{r}_{\mathrm{BGS}} / 100 \%=\frac{\sum_{i=1}^{m} x_{i} / m}{\left(\sum_{i=1}^{m} y_{i}+\sum_{j=1}^{n-m} z_{j}\right) / n}=\frac{\bar{x}}{\left(\frac{m}{n}\right) \bar{y}+\left(\frac{n-m}{n}\right) \bar{z}}
$$

and the new estimator takes the form

$$
\hat{r}_{\text {new }} / 100 \%=\frac{\sum_{i=1}^{m} x_{i} / m}{\sum_{i=1}^{m} y_{i} / m}=\frac{\bar{x}}{\bar{y}} .
$$

An application of the delta method to the expressions (7) and (8) then gives

$$
\operatorname{Var}\left(\hat{r}_{\mathrm{BGS}} / 100 \%\right) \approx \frac{\sigma_{1}^{2}}{m \mu_{2}^{2}}-\frac{2 \mu_{1} \sigma_{12}}{n \mu_{2}^{3}}+\frac{\mu_{1}^{2} \sigma_{2}^{2}}{n \mu_{2}^{4}}
$$

and

$$
\operatorname{Var}\left(\hat{r}_{\text {new }} / 100 \%\right) \approx \frac{\sigma_{1}^{2}}{m \mu_{2}^{2}}-\frac{2 \mu_{1} \sigma_{12}}{m \mu_{2}^{3}}+\frac{\mu_{1}^{2} \sigma_{2}^{2}}{m \mu_{2}^{4}} .
$$

Now suppose that the variability in runs scored is roughly proportional to the number of expected runs scored (i.e. $\sigma_{1} / \sigma_{2} \approx \mu_{1} / \mu_{2}$ ). Then

$$
\begin{aligned}
\operatorname{Var}\left(\hat{r}_{\mathrm{BGS}} / 100 \%\right)-\operatorname{Var}\left(\hat{r}_{\mathrm{new}} / 100 \%\right) & \approx \frac{(n-m) \mu_{1}}{m n \mu_{2}^{3}}\left(2 \sigma_{12}-\frac{\mu_{1} \sigma_{2}^{2}}{\mu_{2}}\right) \\
& \approx \frac{(n-m) \mu_{1}}{m n \mu_{2}^{3}}\left(2 \sigma_{12}-\sigma_{1} \sigma_{2}\right) \\
& >0
\end{aligned}
$$

using the correlation assumption (6). Hence the estimator $\hat{r}_{\text {new }}$ is preferred over $\hat{r}_{\mathrm{BGS}}$ in terms of variability.

To investigate the suitability of assumption (6), we calculated the sample correlation coefficient between $x$ and $y$ for various combinations of $u$ and $w$. Specifically, we chose $u=15,14, \ldots, 6$ to assess the "middle" stages of a match. The corresponding value of $w$ for a particular $u$ is the value for which the sample size $m$ is greatest. The respective sample correlations are $0.45,0.50,0.57,0.62,0.59,0.66,0.58,0.80,0.79$ and 0.82 which tend to exceed 0.5. Hence, there is evidence that assumption (6) is sensible. 
Wickets Lost

\begin{tabular}{c||r|r|r|r|r|r|r|r|r|r} 
Overs Available & 0 & 1 & 2 & 3 & 4 & 5 & 6 & 7 & 8 & 9 \\
\hline 20 & 100.0 & 96.8 & 92.6 & 86.7 & 78.8 & 68.2 & 54.4 & 37.5 & 21.3 & 8.3 \\
19 & 96.1 & 93.3 & 89.2 & 83.9 & 76.7 & 66.6 & 53.5 & 37.3 & 21.0 & 8.3 \\
18 & 92.2 & 89.6 & 85.9 & 81.1 & 74.2 & 65.0 & 52.7 & 36.9 & 21.0 & 8.3 \\
17 & 88.2 & 85.7 & 82.5 & 77.9 & 71.7 & 63.3 & 51.6 & 36.6 & 21.0 & 8.3 \\
16 & 84.1 & 81.8 & 79.0 & 74.7 & 69.1 & 61.3 & 50.4 & 36.2 & 20.8 & 8.3 \\
15 & 79.9 & 77.9 & 75.3 & 71.6 & 66.4 & 59.2 & 49.1 & 35.7 & 20.8 & 8.3 \\
14 & 75.4 & 73.7 & 71.4 & 68.0 & 63.4 & 56.9 & 47.7 & 35.2 & 20.8 & 8.3 \\
13 & 71.0 & 69.4 & 67.3 & 64.5 & 60.4 & 54.4 & 46.1 & 34.5 & 20.7 & 8.3 \\
12 & 66.4 & 65.0 & 63.3 & 60.6 & 57.1 & 51.9 & 44.3 & 33.6 & 20.5 & 8.3 \\
11 & 61.7 & 60.4 & 59.0 & 56.7 & 53.7 & 49.1 & 42.4 & 32.7 & 20.3 & 8.3 \\
10 & 56.7 & 55.8 & 54.4 & 52.7 & 50.0 & 46.1 & 40.3 & 31.6 & 20.1 & 8.3 \\
9 & 51.8 & 51.1 & 49.8 & 48.4 & 46.1 & 42.8 & 37.8 & 30.2 & 19.8 & 8.3 \\
8 & 46.6 & 45.9 & 45.1 & 43.8 & 42.0 & 39.4 & 35.2 & 28.6 & 19.3 & 8.3 \\
7 & 41.3 & 40.8 & 40.1 & 39.2 & 37.8 & 35.5 & 32.2 & 26.9 & 18.6 & 8.3 \\
6 & 35.9 & 35.5 & 35.0 & 34.3 & 33.2 & 31.4 & 29.0 & 24.6 & 17.8 & 8.1 \\
5 & 30.4 & 30.0 & 29.7 & 29.2 & 28.4 & 27.2 & 25.3 & 22.1 & 16.6 & 8.1 \\
4 & 24.6 & 24.4 & 24.2 & 23.9 & 23.3 & 22.4 & 21.2 & 18.9 & 14.8 & 8.0 \\
3 & 18.7 & 18.6 & 18.4 & 18.2 & 18.0 & 17.5 & 16.8 & 15.4 & 12.7 & 7.4 \\
2 & 12.7 & 12.5 & 12.5 & 12.4 & 12.4 & 12.0 & 11.7 & 11.0 & 9.7 & 6.5 \\
1 & 6.4 & 6.4 & 6.4 & 6.4 & 6.4 & 6.2 & 6.2 & 6.0 & 5.7 & 4.4
\end{tabular}

Table 1: The Duckworth-Lewis resource table (Standard Edition) scaled for T20. The table entries indicate the percentage of resources remaining in a match with the specified number of overs available and wickets lost. 


\begin{tabular}{c||r|r|r|r|r|r|r|r|r|r}
\multicolumn{1}{l|}{ Overs Available } & 0 & 1 & 2 & 3 & 4 & 5 & 6 & 7 & 8 & 9 \\
\hline 20 & 100.0 & 98.6 & 96.9 & 93.7 & 83.9 & 73.8 & 60.1 & 45.4 & 29.1 & 18.1 \\
19 & 98.2 & 96.7 & 95.4 & 91.6 & 80.6 & 70.4 & 56.7 & 42.9 & 26.6 & 15.8 \\
18 & 95.3 & 93.4 & 91.9 & 90.4 & 78.5 & 68.3 & 54.6 & 41.3 & 25.2 & 14.5 \\
17 & 91.1 & 88.3 & 85.7 & 82.4 & 77.1 & 66.6 & 52.6 & 40.1 & 24.0 & 13.4 \\
16 & 86.3 & 83.3 & 80.6 & 77.6 & 73.3 & 65.3 & 50.8 & 39.0 & 23.1 & 12.5 \\
15 & 81.6 & 78.5 & 75.9 & 73.0 & 69.3 & 64.4 & 49.0 & 38.1 & 22.1 & 11.7 \\
14 & 77.0 & 73.9 & 71.3 & 68.5 & 65.3 & 61.2 & 47.2 & 37.1 & 21.3 & 11.0 \\
13 & 72.8 & 69.6 & 66.8 & 64.2 & 61.2 & 56.9 & 45.2 & 36.2 & 20.4 & 10.3 \\
12 & 68.4 & 65.3 & 62.7 & 60.1 & 57.1 & 52.8 & 43.1 & 35.4 & 19.5 & 9.7 \\
11 & 64.0 & 60.9 & 58.3 & 55.8 & 52.7 & 48.5 & 40.7 & 34.6 & 18.5 & 9.1 \\
10 & 59.2 & 56.1 & 53.6 & 51.3 & 48.2 & 43.9 & 38.3 & 33.9 & 17.6 & 8.5 \\
9 & 54.7 & 51.7 & 49.2 & 46.9 & 43.8 & 40.0 & 35.9 & 33.2 & 16.6 & 7.8 \\
8 & 50.1 & 47.2 & 44.7 & 42.5 & 39.7 & 36.2 & 32.7 & 29.1 & 15.5 & 7.1 \\
7 & 45.6 & 42.5 & 40.2 & 38.0 & 35.5 & 32.2 & 28.8 & 24.2 & 14.2 & 6.4 \\
6 & 40.5 & 37.5 & 35.3 & 33.2 & 31.1 & 28.4 & 24.8 & 20.4 & 12.4 & 5.5 \\
5 & 32.8 & 31.0 & 29.3 & 27.6 & 25.8 & 23.7 & 20.8 & 16.9 & 10.7 & 4.6 \\
4 & 29.1 & 26.5 & 24.8 & 23.1 & 21.3 & 19.3 & 16.9 & 13.5 & 8.8 & 3.8 \\
3 & 23.3 & 20.1 & 18.9 & 17.6 & 16.4 & 14.8 & 12.9 & 10.1 & 6.7 & 2.8 \\
2 & 17.3 & 14.1 & 13.2 & 12.2 & 11.2 & 10.1 & 8.7 & 6.9 & 4.4 & 1.8 \\
1 & 10.0 & 6.1 & 5.7 & 5.3 & 4.8 & 4.3 & 3.7 & 2.9 & 1.9 & 0.8
\end{tabular}

Table 2: A resource table for T20 based on a slight modification of the methods of BGS as described in section 3. The table entries indicate the percentage of resources remaining in a match with the specified number of overs available and wickets lost. 
Wickets Lost

\begin{tabular}{c||r|r|r|r|r|r|r|r|r|r} 
Overs Available & 0 & 1 & 2 & 3 & 4 & 5 & 6 & 7 & 8 & 9 \\
\hline 20 & 100.0 & 97.6 & 97.3 & 86.7 & 78.8 & 68.2 & 54.5 & 38.4 & 22.0 & 9.5 \\
19 & 98.5 & 97.3 & 97.1 & 85.2 & 76.6 & 66.6 & 53.5 & 37.4 & 21.6 & 9.4 \\
18 & 97.5 & 95.9 & 94.0 & 84.8 & 75.3 & 65.0 & 52.6 & 37.0 & 21.3 & 9.3 \\
17 & 95.3 & 93.0 & 91.4 & 84.4 & 75.1 & 63.4 & 51.5 & 36.5 & 21.1 & 9.2 \\
16 & 91.5 & 87.8 & 84.2 & 80.3 & 75.0 & 62.9 & 50.4 & 36.1 & 21.0 & 9.1 \\
15 & 86.8 & 82.8 & 80.9 & 75.6 & 73.7 & 61.9 & 49.1 & 35.7 & 20.8 & 9.0 \\
14 & 77.2 & 73.6 & 70.7 & 68.7 & 63.9 & 58.8 & 47.7 & 35.2 & 20.7 & 8.9 \\
13 & 74.6 & 69.0 & 64.6 & 60.5 & 56.5 & 53.0 & 46.1 & 34.8 & 20.6 & 8.8 \\
12 & 64.7 & 63.0 & 58.9 & 56.8 & 53.9 & 52.7 & 44.3 & 34.5 & 20.4 & 8.7 \\
11 & 61.4 & 58.5 & 52.0 & 50.0 & 47.6 & 43.3 & 43.1 & 33.5 & 20.2 & 8.6 \\
10 & 55.1 & 53.4 & 48.8 & 46.5 & 43.8 & 40.2 & 38.1 & 32.4 & 20.0 & 8.5 \\
9 & 52.6 & 47.0 & 41.7 & 40.5 & 38.2 & 36.3 & 34.3 & 29.1 & 19.7 & 8.4 \\
8 & 47.3 & 41.9 & 37.8 & 35.6 & 33.9 & 31.3 & 29.2 & 28.2 & 19.2 & 8.3 \\
7 & 42.4 & 40.2 & 33.4 & 31.7 & 29.2 & 27.7 & 26.4 & 24.6 & 18.5 & 8.2 \\
6 & 37.2 & 34.9 & 29.8 & 27.2 & 26.0 & 24.5 & 21.7 & 19.9 & 17.8 & 8.1 \\
5 & 32.4 & 32.1 & 24.6 & 24.1 & 22.3 & 21.6 & 19.0 & 17.4 & 17.2 & 8.0 \\
4 & 27.9 & 27.5 & 21.1 & 19.4 & 18.3 & 16.8 & 16.3 & 14.2 & 10.8 & 7.7 \\
3 & 20.2 & 19.9 & 16.8 & 16.2 & 15.2 & 13.9 & 12.8 & 10.7 & 9.8 & 5.8 \\
2 & 14.8 & 14.6 & 10.5 & 10.2 & 9.5 & 9.1 & 8.9 & 8.3 & 6.9 & 4.8 \\
1 & 8.8 & 8.7 & 7.8 & 6.4 & 6.2 & 5.5 & 5.2 & 4.7 & 4.5 & 2.9
\end{tabular}

Table 3: A new resource table for T20 based on the the methods as described in section 4. The table entries indicate the percentage of resources remaining in a match with the specified number of overs available and wickets lost. 


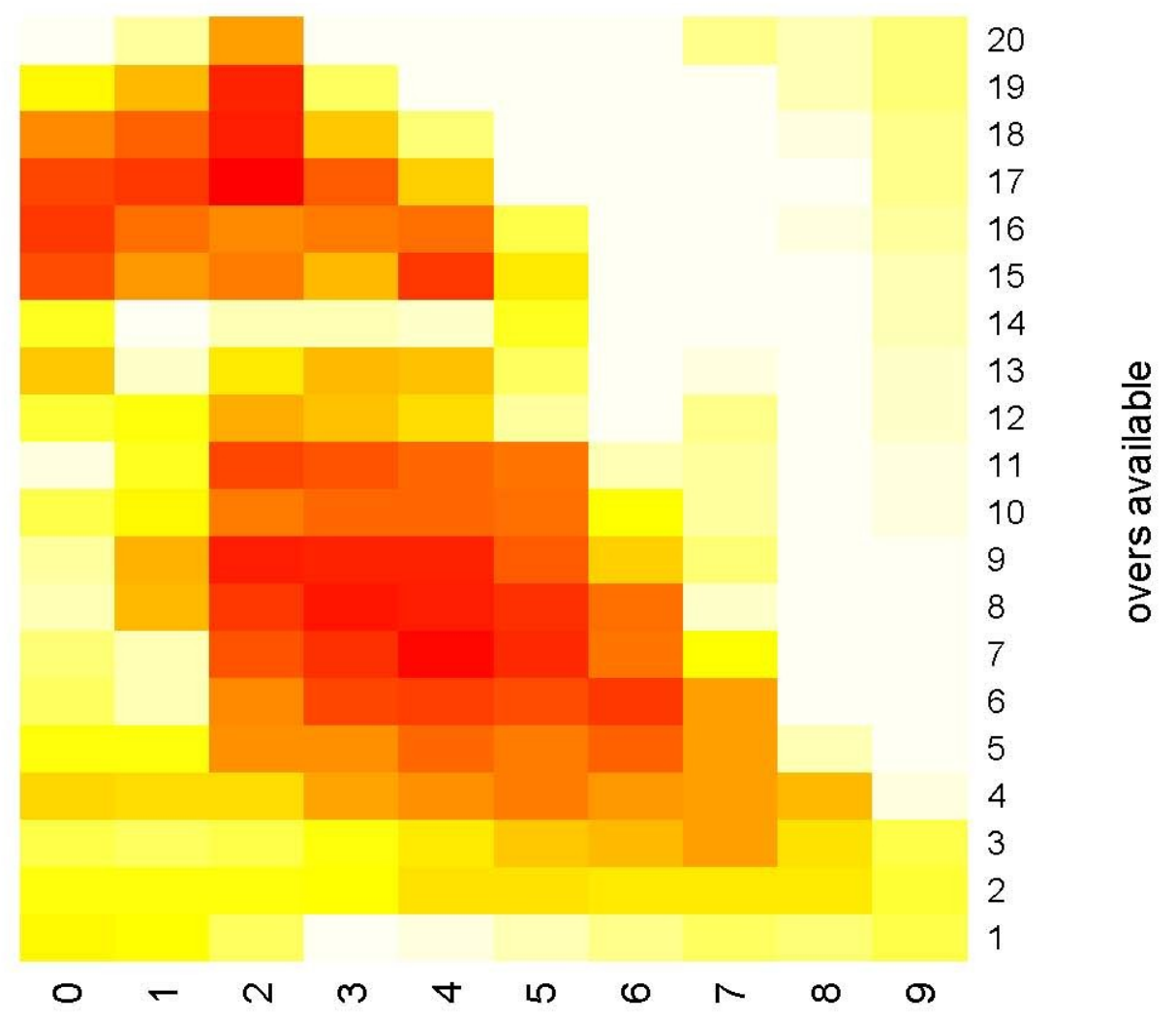

wickets lost

Figure 1: Heat map of the absolute differences between the Duckworth-Lewis resource table (Table 1) and the new resource table (Table 3) described in Section 4. Darker shades indicate larger differences. 